\title{
INFLUENCIA DE LA MOTIVACION LABORAL EN EL CLIMA ORGANIZACIONAL DE DISFRUTA - HUANCAYO
}

\author{
THE INFLUENCE OF THE LABOR MOTIVATION IN THE WORK ENVIRONMENT \\ OF DISFRUTA - HUANCAYO \\ Jessica Patrick Poves Varillas, Sergio Belisario Gamarra Pascual.
}

\begin{abstract}
RESUMEN
La presente investigación titulada "Influencia de la Motivación Laboral en el Clima Organizacional”, permitió evaluar la motivación laboral de DisFruta - Huancayo; con respecto a un Marco Referencial que integre: Planteamientos Teóricos referentes a este tipo de estudio, las condiciones del Contexto Interno de influencia y las Normas que rigen la misma.

De tal manera, que se realizaron observaciones previas utilizando la técnica de la encuesta, utilizado como instrumento para recopilación de datos de campo con un Cuestionario, recurriendo como informantes a las colaboradoras de DisFruta-Huancayo, para diagnosticar los problemas, y después se volvió a utilizar la técnica de la encuesta para continuar con la investigación , también se utilizo la técnica de la entrevista, utilizado como instrumento para recopilación de datos de campo con una Guía de entrevistas, recurriendo como informante a la responsable de DisFruta-Huancayo.

El Modelo Aplicativo, basado en gran parte en la Teoría de Maslow, se dividió en tres partes, para lograr sus objetivos; la primera parte se capacitó a las colaboradoras de DisFruta - Huancayo, en vista de que existen empirismos aplicativos, la segunda parte concierne básicamente la Teoría de Maslow, donde se evalúa las necesidades de las colaboradoras, y se encuentra que están insatisfechas, para ello previamente se analizó la personalidad y las actitudes, para así poder entender mejor a la colaboradora, en base a todos los datos recogidos, es que se aplica acciones de solución, que dan como resultado la satisfacción de las colaboradoras , por último, la tercera parte como ya se hizo mención, se basa en la re-evaluación de la empresa, en cuanto a los conocimientos vertidos en la capacitación , y una re-evaluación de sus necesidades.

Llegando a la conclusión que la motivación laboral en DisFruta - Huancayo, fue afectada por Empirismos Aplicativos, Carencias y Deficiencias, que influyeron negativamente al clima organizacional de la empresa; por no respetar o trasmitir mal las políticas laborales para lograr los objetivos, por desconocer o aplicar mal algunos planteamientos teóricos referentes a este tipo de investigación, o porque no se ajusto adecuadamente a las condiciones de su contexto interno. Problemas que se resolvieron con la Intervención Metodológica, logrando de esta manera mejorar la motivación laboral de las colaboradoras de DisFruta - Huancayo, que se vera reflejado en el clima organizacional.

Así como también se recomienda, que se continúe con las acciones realizadas en la investigación, en busca de una mejora continua para la empresa, también se recomienda que se incorpore personal del genero masculino, a fin de que exista mayor espacios de diálogo, armonía y equilibrio, logrando así que la motivación siempre sea la adecuada, aquella que influenciara en el clima organizacional.
\end{abstract}

Palabras clave: motivación laboral, clima organizacional, Teoría de Maslow, personalidad.

\section{ABSTRACT}

This research work, named "Influence of the work motivation in the organizational environment" let evaluate the DisFruta-Huancayo work motivation; based in a theoretical background that integrates: theoretical issues, internal context conditions influences and the laws that govern this context.

Previous observations were done using the poll technique, using a questionnaire and having as informers the DisFruta-Huancayo workers, in order to diagnose the problems. The interview technique was also used.

The applied model based in Maslow theory is divided in three parts, in order to reach its objectives: in the first part DisFruta Huancayo workers were trained; in the second part the workers needs were evaluated, and it was found that the workers were not satisfied based on a previous analysis that take into account the attitudes and personalities of the workers for a better understanding, based on the collected data actions are applied, these actions generate satisfaction in the worker; at last, in the third part, the enterprise is evaluated based on the training knowledge and the needs reevaluation.

It can be stated as a conclusion that work motivation in DisFruta workers was affected by applied empirics, lacks and deficiencies had a negative influence in the enterprise organizational environment. Because they do not respect or make a bad transmission of the work policies to reach the objectives, they do not know or are bad applied some theoretical issues related to this kind of research, or because it did not adequate to the conditions of the intern context. Problems that were solved with the methodological intervention, achieving in this way to improve the work motivation of the DisFruta Huancayo Workers, and this fact will be reflected in the organizational environment.

Also is recommended, that the actions derived from this research could be maintained, in order to look for a continuous improvement of the Enterprise, besides, is recommended that it could be incorporated male gender workers, for furthering more dialog, harmony and equilibrium. In this way the adequate motivation that influences the organizational environment will also be improved.

Key words: work motivation, organizational environment, Maslow theory, personality.

\section{PLANTEAMIENTO DEL PROBLEMA}

Los nuevos centros comerciales han aparecido como una gran ola en el Perú, expansiva e impetuosa, que es el crecimiento económico más fuerte que experimenta éste país, que se refleja en la mejora de los niveles de consumo de la población. El Perú, con algo más de 28 millones de habitantes, inició el 2010 con una veintena de centros comerciales en funcionamiento, en tanto que en Lima y en provincias se construyó otra veintena de ellos. Según la Asociación de Centros Comerciales y de Entretenimiento del Perú (ACCEP), la inversión en centros comerciales en el 2010 fue de US\$ 816 millones de dólares aproximadamente.

Y se proyecta que a fines del 2011 , habrá unos 40 centros comerciales y, a éste ritmo, en cinco años el país contará con unos cien centros comerciales si no es que más, sostuvo el presidente de la ACCE, Juan Calle.

En Lima, por el alto nivel de sus ventas, los centros comerciales están liderados por Jockey Plaza y Plaza San Miguel, ubicados en las zonas este y oeste de la capital, respectivamente. Pero también compiten por el liderazgo Mega Plaza Norte y el Real Plaza-Centro Cívico. 
Mientras tanto, dos grupos de inversionistas peruanos, Estructura y E. Wong, construyen en Lima sus respectivos centros comerciales. Estructura tiene a su cargo el proyecto del centro comercial Las Malvinas, en el corazón histórico de la capital, con 30.000 metros cuadrados, 915 locales y 26 millones de dólares en inversión. $Y$ el proyecto de $E$. Wong se desarrolla sobre un terreno de 169.000 metros cuadrados en Santa María del Mar, un distrito ubicado en el litoral sur de Lima.

En provincias, Piura y Arequipa lideran la construcción de nuevos centros comerciales, en tanto que el puerto de Chimbote verá el surgimiento de seis de esos centros en los próximos años. Piura, a 982 kilómetros de Lima, cerrará el año con tres nuevos centros comerciales: Open Plaza, Real Plaza y Plaza de la Luna, además de la ampliación de Plaza del Sol, en los cuales se calcula que se ha invertido US\$ 130 millones de dólares. Algo parecido ocurrirá en Arequipa, donde han surgido este año Real Plaza, Parque Lambramani y Mall Aventura Plaza cuya construcción, en conjunto, se calcula en US\$ 169 millones de dólares americanos.

Y por último la ciudad Incontrastable, Huancayo, es sin duda alguna un nuevo mercado interesante para los inversionistas, así lo demuestra el Real Plaza, centro comercial, proyecto del grupo Interbank que demandó una inversión de S/.90 millones de nuevos soles, está construido sobre un terreno de 40.000 $\mathrm{m}^{2}$, sobre lo que fue una antigua estación del Ferrocarril Central. Tiene un Plaza Vea y siete salas de Cineplanet, entre otros importantes establecimientos.

Las ventas de los centros comerciales sumaron para el 2010, US\$ 3.500 millones de dólares americanos, mientras que para el 2011, según cálculos de la ACCEP, llegarían a los US\$ 4.000 millones de dólares americanos, impulsadas por el mayor consumo y la apertura de nuevos locales en Lima y provincias.

Juan Calle (ACCE) recordó que en 2008 las ventas de estos establecimientos sumaron, US\$ 2.290 millones de dólares americanos, lo que significó un crecimiento de 30 por ciento respecto al año anterior. En el 2009 las ventas fueron de, US\$ 2,360 millones de dólares aproximadamente. Y en el 2010 las ventas ascendieron a, US\$ 3.500 millones millones de dólares, mientras que para el 2011 se espera que el monto aumente a US\$ 4.000 millones de dólares. En opinión del empresario, el auge de los centros comerciales en el Perú tiene que ver con el crecimiento económico del país, el impulso de la minería, la creación de nuevas fuentes de trabajo en provincias, etcétera, todo lo cual ha devenido en una mayor capacidad de consumo de la población.

El grupo chileno Parque Arauco, asociado con el grupo Wiese del Perú son promotores de Mega Plaza Norte Lima, Strip Center y Mega Express Villa Lima. Los centros comerciales operados sólo por Parque Arauco son Larcomar Lima y Parque Lambramani en Arequipa.

El grupo chileno tiene en proyecto el Parque El Golf Lima, Mega Plaza Chimbote y Parque El Agustino Lima. El grupo peruano $\mathrm{E}$ Wong tiene a su cargo los centros comerciales Plaza Norte y Santa María del Mar, mientras que proyecta la construcción de nuevos centros en provincias.

Los nuevos centros comerciales son espacios muy modernos, de arquitectura y diseño dinámicos, y equipados con los últimos adelantos de la tecnología, donde la tranquilidad, la variedad de servicios y facilidades que ofrecen los centros comerciales, cuando se hacen compras, han sido, sin duda alguna, los motivos suficientes para que éstos crezcan sostenidamente. A diferencia de los centros comerciales antiguos, los nuevos no venden sus espacios, sino que los alquilan, y su funcionamiento, regido por una serie de exigencias de calidad, está a cargo de una administración centralizada.

\section{METODOLOGÍAS EMPLEADAS}

\subsection{MOTIVACION.}

Según Chiavenato, la motivación está constituida por todos los factores capaces de provocar, mantener y dirigir la conducta hacia un objetivo.

Hoy en día es un elemento importante en la administración de personal por lo que se requiere conocerlo, y más que ello, dominarlo, sólo así la empresa estará en condiciones de formar una cultura organizacional sólida y confiable.

Así mismo menciona que la motivación también es considerada como el impulso que conduce a una persona a elegir y realizar una acción entre aquellas alternativas que se presentan en una determinada situación. En efecto, la motivación está relacionada con el impulso, porque éste provee eficacia al esfuerzo colectivo orientado a conseguir los objetivos de la empresa, por ejemplo, y empuja al individuo a la búsqueda continua de mejores situaciones a fin de realizarse profesional y personalmente, integrándolo así en la comunidad donde su acción cobra significado.

\subsection{LA MOTIVACION Y LA PERSONALIDAD.}

a) PERSONALIDAD.

Al tratarse de un concepto básico dentro de la psicología, a lo largo de la historia ha recibido numerosas definiciones, además de las conceptualizaciones más o menos intuitivas que ha recibido. Algunos autores han clasificado estas definiciones en grupos.

Según Henry Murray, la personalidad puede sintetizarse como el conjunto de características o patrón de sentimientos y pensamientos ligados al comportamiento, es decir, los pensamientos, sentimientos, actitudes y hábitos y la conducta de cada individuo, que persiste a lo largo del tiempo frente a distintas situaciones distinguiendo a un individuo de cualquier otro haciéndolo diferente a los demás. La personalidad persiste en el comportamiento de las personas congruentes a través del tiempo, aun en distintas situaciones o momentos, otorgando algo único a cada individuo que lo caracteriza como independiente y diferente. Ambos aspectos de la personalidad, distinción y persistencia, tienen una fuerte vinculación con la construcción de la identidad, a la cual modela con características denominadas rasgos o conjuntos de rasgos que, junto con otros aspectos del comportamiento, se integran en una unidad coherente que finalmente describe a la persona. Ese comportamiento tiene una tendencia a repetirse a través del tiempo de una forma determinada, sin que quiera decir que esa persona se comporte de modo igual en todos los casos. Es decir, la personalidad es la forma en que pensamos, sentimos, nos comportamos e interpretamos la realidad, mostrando una tendencia de ese comportamiento a través del tiempo, que nos permite afrontar la vida y mostrarnos el modo en que nos vemos a nosotros mismos y al mundo que nos rodea. Nos permite reaccionar ante ese mundo de acuerdo al modo de percepción, retro-alimentando con esa conducta en nuestra propia personalidad. Cada persona al nacer ya tiene su propia personalidad con ciertas características propias, que con el paso del tiempo más el factor ambiental y las circunstancias es como se definirá esa persona. La personalidad será fundamental para el desarrollo de las demás habilidades del individuo y para la integración con grupos sociales.

\section{b) FACTORES DETERMINANTES DE LA} PERSONALIDAD.

Robbins menciona los factores determinantes de la personalidad y estos son: 
- Factores Orgánicos.- Esta refiere a aquellos factores que determinaron en la concepción. La estatura física, el atractivo facial, el género, el temperamento, la composición de músculos y reflejos, el nivel de energía y los ritmos biológicos son características que por lo general se considera que están, ya sea completa o sustancialmente, influenciadas por los padres. Esto es, por su arreglo biológico, sociológico y psicológico inherente. El método de la herencia sostiene que la explicación última de la personalidad del individuo es la estructura molecular de los genes, localizados en los cromosomas.

- Factores Psicológicos o Personales.- Entre los factores que ejercen presiones en la formación de la personalidad, están la cultura en la cual se creció, los primeros aprendizajes, las normas de nuestra familia y los grupos sociales y otras influencias que experimentamos. El ambiente al que se es expuesto juega un papel sustancial en el moldeamiento de la personalidad. Ejemplo, la cultura establece las normas las actitudes y los valores que pasan de una generación a otra y crea consistencias con el tiempo. Una ideología que se nutre con intensidad en una cultura podría influenciar moderadamente en otra. Ejemplo, los americanos han tenido los temas de la industrialización, el éxito, la competencia, la independencia y la ética de trabajo de los protestantes, constantemente implantados a través de los libros, el sistema escolar, la familia y los amigos. Los americanos, como resultado de ello, tienden a ser ambiciosos y empeñosos en relación con los individuos que crecieron en culturas que han enfatizado el llevarse bien con los demás, la cooperación y la prioridad de la familia sobre el trabajo y la carrera.

Una cuidadosa consideración de los argumentos que favorecen tanto la herencia o el ambiente como determinante principal de la personalidad fuerza y la conclusión de que ambos son importantes. La herencia establece los parámetros o límites exteriores, pero el potencial completo de un individuo será determinado por cuán bien él o ella se ajuste a las demandas y requerimientos del trabajo.

- Factores Ambientales.- tercer factor, influye en los efectos de la herencia y el ambiente de la personalidad. La personalidad de un individuo, aunque generalmente estable y consistente, cambia en situaciones diferentes. Las distintas demandas de diversas situaciones competen a 4 aspectos diferentes de la personalidad. No se debería, por tanto, observar los patrones de personalidad en forma aislada. Parece lógico suponer que las situaciones influirán en la personalidad del individuo, pero en un plan de clarificación claro que se mostrará el impacto de varios tipos de situaciones, que se ha eludido hasta ahora. Aparentemente todavía no se esta cerca de desarrollar un sistema para clarificar situaciones que pudieran estudiarse de manera sistemática. Sin embargo, se sabe que ciertas situaciones tienen mayor influencia en la personalidad. Lo que es taxonómicamente de interés es que las situaciones parecen diferir sustancialmente en las restricciones que imponen sobre el comportamiento. Algunas situaciones, por ejemplo, la iglesia, las entrevistas de empleos, restringen muchos comportamientos, otras situaciones, por ejemplo, un día de campo en un parque público, restringen relativamente pocos.

\subsection{TEORIA PSICOANALITICA DE SIGMUND FREUD.}

El psicoanálisis es una teoría sobre los procesos psíquicos inconscientes, que presenta una concepción ampliada de la sexualidad, de sus relaciones con el acontecer psíquico y su reflejo en lo sociocultural. El supuesto de que existen los procesos, fenómenos y mecanismos psíquicos inconscientes junto al papel de la sexualidad y del llamado complejo de Edipo, que resulta en una diferenciación entre instinto y pulsión; así como la aceptación de la teoría de la represión y el papel de la resistencia en el análisis constituyen para Freud los pilares fundamentales de su edificio teórico, al punto que sostiene que «quien no pueda admitirlos todos no debería contarse entre los psicoanalistas».3

Esta definición de Freud ha tenido amplia aceptación entre los psicoanalistas, así como también la necesidad de la transmisión del saber a través del análisis didáctico y los análisis de control o supervisados. Esta aceptación o identificación con los pilares centrales de la teoría y el método, se dio en medio de controversias. Refiriéndose a la definición de Freud de 1922.

\subsection{TIPOS DE PERSONALIDAD}

Meyer Friedman, propone tres tipos de personalidad de las personas, para entenderlas y juzgarlas mejor, a continuación los tipos de personalidad:

- Tipo de Personalidad A:

Este tipo de personas deben de relajarse y tomarse las cosas con más filosofía, pues tiene mayor peligro de padecer enfermedades coronarias, problemas psicosomáticos y estados de ansiedad. Su características principales son la; Inseguridad y baja autoestima, una impaciencia extrema que causa irritación y exasperación y por ultimo una excesiva hostilidad que puede ser activarse con incidentes menores.

- Tipo de Personalidad B:

Este tipo de personas son bastante menos vulnerables a sufrir ansiedad que otras personas, no se muestran ambiciosos ni dominantes, dejan que las cosas sigan su cauce sin preocuparse en exceso. No quiere decir que nunca se muestren nerviosos o angustiados si la situación le desborda, pero en general tienen un temperamento templado.

- Tipo de Personalidad Mixto:

El carácter de este tipo de personas es un equilibrio entre la personalidad A y la B. Dentro de estos parámetros es donde se encuentra la mayoría de personas. Se activa lo suficiente para ser productivo y eficiente en el trabajo o estudios, pero sabe mantener la calma en las situaciones que así lo requieren, aunque en ocasiones también se sienten nerviosos.

\subsection{CLIMA ORGANIZACIONAL}

Según Chiavenato, éste concepto se refiriere concretamente a todos aquellos elementos relacionados con los procesos de gestión, ya sean formales o informales (soportados, por tanto por la cultura de la compañía, en los procedimientos establecidos o en la interpretación y uso que de éstos hagan los líderes de los equipos) que influyen positiva o negativamente en el trabajo.

Con ellos se deja fuera todos los elementos del entorno físico (ergonomía) porque no están causados por comportamientos organizativos.

Esto permite concretar el clima, y las mejoras que puedan suceder a su medición y análisis, en aquellos elementos que tienen al mismo tiempo interés para el profesional (mejoran su percepción sobre la organización) y para la empresa (mejoran el rendimiento de sus equipos).

a) Dimensiones que se deben considerar a la hora de analizar el clima organizacional:

Chiavenato, menciona las dimensiones que se deben de considerar a la hora de analizar el Clima Organizacional y ellos son: 
Flexibilidad: el grado en que los individuos perciben restricciones o flexibilidad en la organización, es decir, hasta qué punto las reglas, políticas, procedimientos o prácticas son innecesarias o interfieren con la ejecución del trabajo. También, refleja la medida en que se aceptan nuevas ideas.

Responsabilidad: el grado en que los individuos perciben que se les delega autoridad y pueden desempeñar sus trabajos sin tener que consultar constantemente al supervisor y la medida en que sienten que la responsabilidad del resultado está en ellos.

Recompensas: el grado en que los individuos perciben que son reconocidos y recompensados por un buen trabajo y que esto se relaciona con diferentes niveles de desempeño.

Claridad: el grado en que los individuos perciben que las metas, los procedimientos, las estructuras organizativas y el flujo de trabajo están claramente definidos, de manera que todo el mundo sabe que tiene que hacer y a relación que estos guarda con los objetivos generales de la organización.

Espíritu de equipo: el grado en la gente se siente orgullosa de pertenecer a la organización y sienten que todos están trabajando hacia aun objetivo común.

b) Principales factores que determinan el Clima Organizacional:

Así mismo Chiaventato nos menciona los principales factores que determinan el Clima Organizacional, y estos son:

Condiciones físicas: Las condiciones físicas contemplan las características medioambientales en las que se desarrolla el trabajo: la iluminación, el sonido, la distribución de los espacios, la ubicación (situación) de las personas, los utensilios, etcétera. Por ejemplo: un medio con luz natural, con filtros de cristal óptico de alta protección en las pantallas de los ordenadores, sin papeles ni trastos por el medio y sin ruidos, facilita el bienestar de las personas que pasan largas horas trabajando y repercute en la calidad de su labor. Se ha demostrado científicamente que las mejoras hechas en la iluminación aumentan significativamente la productividad.

Liderazgo: Mide la capacidad de los líderes para relacionarse con sus colaboradores. Un liderazgo que es flexible ante las múltiples situaciones laborales que se presentan, y que ofrece un trato a la medida de cada colaborador, genera un clima de trabajo positivo que es coherente con la misión de la empresa y que permite y fomenta el éxito.

El grado de madurez: el respeto, la manera de comunicarse unos con otros, la colaboración o la falta de compañerismo, la confianza, todo ello son aspectos de suma importancia. La calidad en las relaciones humanas dentro de una empresa es percibida por los clientes.

\subsection{NECESIDADES HUMANAS:}

Antes de entrar al tema de necesidades es necesario mencionar a la motivación la cual sucede en el interior de la persona producto de una necesidad, por lo que no es observable, pero sí distinguible en la conducta que desencadena.

Es definida según Boeree (1970) como el proceso por el cual una necesidad insatisfecha mueve a una persona en cierta dirección para lograr un objetivo que satisface dicha necesidad. Es un impulso que inicia, guía y mantiene el comportamiento hasta alcanzar la meta u objetivo deseado. De acuerdo a esta definición, se encuentran tres elementos en la motivación, ilustrando el proceso motivacional de la siguiente manera:

$1^{\circ}$ Un deseo o necesidad interior.

$2^{\circ}$ Una meta u objetivo que debe ser logrado.

$3^{\circ}$ Una estrategia para lograr el objetivo. (Ver figura $\left.N^{\circ} 1\right)$

FIGURA No 1

Proceso Motivacional

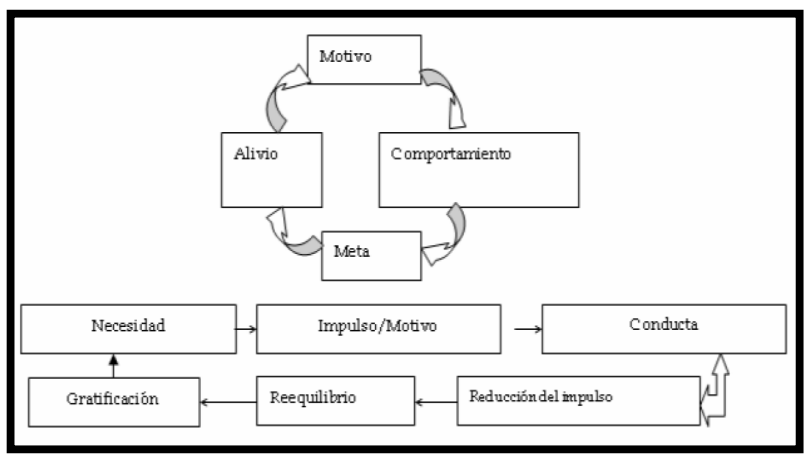

Tomado de: Robbins, Stephen P. (1994).Comportamiento Organizacional. Edición. 6ta Editorial. Prentice Hall Lugar. México.

\subsection{TEORIA DE LAS NECESIDADES HUMANAS.}

A. Necesidades Fisiológicas: Las necesidades fisiológicas son satisfechas mediante comida, bebidas, sueño, refugio, aire fresco, una temperatura apropiada. Si todas las necesidades humanas dejan de ser satisfechas entonces las necesidades fisiológicas se convierten en la prioridad más alta.

B. Necesidades de Seguridad: Para Maslow, son dirigidas a la autoconservación buscando un medio donde no peligre su seguridad.

Éstas se refieren a los requerimientos del organismo de poseer un mundo ordenado, estable y predecible. También llamadas de protección, son las que satisfechas las necesidades fisiológicas empiezan a dominar y motivar la conducta buscando la protección contra el peligro, las amenazas y carencias.

Se han realizado investigaciones sobre la relación entre la inseguridad laboral causada por amenaza de desempleo y su afectación en la salud de los empleados, encontrando que intervienen una gran cantidad de aspectos de salud y de personalidad que varían situacionalmente por lo que esta conjetura no ha encontrado estricta correlación.

C. Necesidades Sociales y de pertenencia: Están relacionadas con el desarrollo afectivo del individuo, son las necesidades de:

Se satisfacen mediante las funciones de servicios y prestaciones que incluyen actividades deportivas, culturales y recreativas. El ser humano por naturaleza siente la necesidad de relacionarse, ser parte de una comunidad, de agruparse en familias, con amistades o en organizaciones sociales. Entre estas se encuentran: la amistad, el compañerismo, el afecto y el amor. Estas se forman a partir del esquema social.

D. Necesidades de Estimación o Ego: Maslow describió dos tipos de necesidades de estima, una alta y otra baja.

- La estima alta concierne a la necesidad del respeto a uno mismo, e incluye sentimientos tales como confianza, competencia, maestría, logros, independencia y libertad.

- La estima baja concierne al respeto de las demás personas: la necesidad de atención, aprecio, reconocimiento, reputación, estatus, dignidad, fama, gloria, e incluso dominio. 
La merma de estas necesidades se refleja en una baja autoestima y el complejo de inferioridad.

E. Necesidades de Autorrealización o Autoactualización: Este último nivel es algo diferente y Maslow utilizó varios términos para denominarlo: «motivación de crecimiento», «necesidad de ser» y «autorrealización».

Son las necesidades más elevadas, se hallan en la cima de la jerarquía, y a través de su satisfacción, se encuentra un sentido a la vida mediante el desarrollo potencial de una actividad. Se llega a ésta cuando todos los niveles anteriores han sido alcanzados y completados, al menos, hasta cierto punto.

FIGURA No 2

Pirámide de la Jerarquización de Necesidades

\begin{tabular}{|l|l|}
\hline \multicolumn{2}{|l|}{} \\
\hline $\begin{array}{l}\text { Autorrealización } \\
\text { Autoactualización) }\end{array}$ & Autoexpresión, independencia, competencia, oportunidad. \\
\hline Estima & $\begin{array}{l}\text { Reconocimiento, responsabilidad, sentimiento de cumplimiento, prestigio, } \\
\text { status, fama. }\end{array}$ \\
\hline $\begin{array}{l}\text { Sociales } \\
\text { (Pertenencia) }\end{array}$ & Compañerismo, aceptación, pertenencia, trabajo en equipo, anvistad. \\
\hline Seguridad & Seguridad, estabilidad, evitar los daños fisicos, evitar los riesgos. \\
\hline Fisiológicas & Alimento, vestido, confort, instinto de conservación. \\
\hline \multicolumn{2}{c}{ Necesidades de Déficit } \\
\hline
\end{tabular}

Tomado de: Rafael Gautier y George Boeree. Libro: Teoría de la Personalidad: una selección de los mejores autores del siglo XX. Editorial: UNIBE Año: 2005

\section{DESARROLLO}

\subsection{DIAGNÓSTICO DEL PROBLEMA}

En DisFruta - Huancayo, se identificaron tres problemas que son; Empirismos Aplicativos, Carencias en la identificación del equipo humano y Deficiencias en las Actividades Laborales.

\subsection{MODELO APLICATIVO}

Realmente, la motivación es la mejor arma para potenciar el rendimiento. Sin embargo, alcanzar la motivación de los empleados requiere una labor compleja de análisis de necesidades (no a todos les motivan las mismas cosas).

Es por ello que la programación intenta casar las expectativas individuales con las necesidades de la empresa, evaluación y, sobre todo, comunicación (el empleado busca sentirse comprendido y escuchado por la empresa).

A continuación se presenta la figura $\mathrm{N}^{\circ} 3$, sobre el Modelo Aplicativo, el presente esta dividido en tres partes;

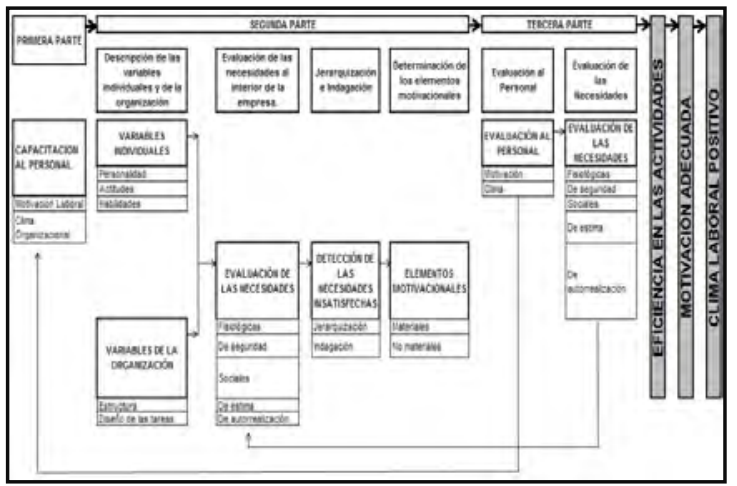

FIGURA No 3

\section{MODELO APLICATIVO}

Basado en: Rafael Gautier y George Boeree. Libro: Teoría de la Personalidad: una selección de los mejores autores del siglo XX. Editorial: UNIBE Año: 2005 Elaboración: Propia

A. Primera Parte: En vista de que existen Empirismos Aplicativos, se capacitará al personal de la empresa en temas como; Motivación Laboral, el Clima Organizacional y Satisfacción Laboral.

B. Segunda Parte: Como se pudo evidenciar en el Capitulo I, se halla Carencias en la identificación del equipo humano, para ello se generará un programa que busque dar una solución, el mismo que se divide en cuatro pasos:

- Paso No 1: Primero se empezará conociendo a la persona, aplicando un Test de personalidad, seguido por una encuesta sobre actitudes y habilidades. Después, basado en el organigrama de la empresa, se diseñarán tareas a todo el personal, a fin de buscar mayor eficiencia en las actividades.

- Paso No 2: Se evaluará las necesidades del personal, considerando la Teoría de necesidad de Abraham Maslow.

- Paso N03: Una vez detectadas las necesidades insatisfechas, se procederá a jerarquizar e indagar los motivos de la insatisfacción.

- Paso № 4: Después de jerarquizar e indagar los motivos de las necesidades insatisfechas, se procederá a dar solución, brindando elementos motivacionales, tanto materiales como no materiales.

C. Tercera Parte: En esta tercera parte de procederá a realizar nuevamente una evaluación y revisión tanto de la primera y segunda parte:

- Paso No 1: Se procede a evaluar al personal, en los temas de Motivación Laboral, el Clima Organizacional, ya que anteriormente fueron capacitados. (Primera Parte)

- Paso No 2: Se realiza una segunda evaluación de las necesidades al personal, para saber si ha mejorado el nivel de satisfacción de sus necesidades.

\section{EVALUACIÓN DEL MODELO APLICATIVO.}

Después de que se realizo la capacitación al personal en temas como; Motivación Laboral y Clima Organizacional, se realizo un test a modo de conocer el tipo de personalidad de las colaboradoras, a continuación los resultados.

FIGURA $\mathrm{N}^{\circ} 4$

Distribución porcentual del tipo de personalidad que poseen las colaboradoras de DisFruta Huancayo

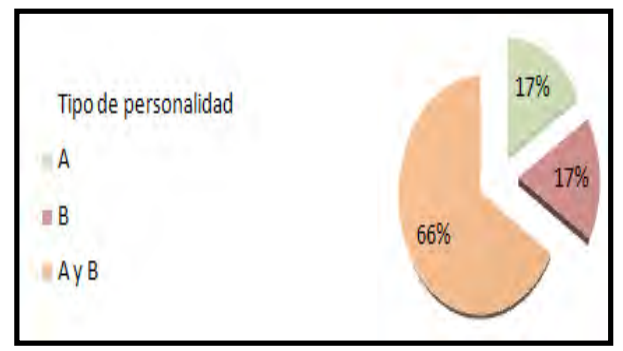

Fuente: DisFruta - Huancayo. Elaboración: Propia

Se puede apreciar que el $66 \%$ de las colaboradoras tienen un tipo de personalidad mixto, aquel tipo de personalidad adecuado, mientras que un $17 \%$ muestra un tipo de personalidad de Tipo A y $\mathrm{B}$, el mismo porcentaje para ambos casos. De esta manera se pudo conocer el tipo de personalidad, que servirá para un mejor análisis. 
A continuación se muestra el Figura $N^{\circ} 5$ sobre la Distribución porcentual de las actitudes que poseen las colaboradoras de DisFruta - Huancayo

FIGURA No 5

Distribución porcentual sobre las actitudes que posee el personal de DisFruta Huancayo

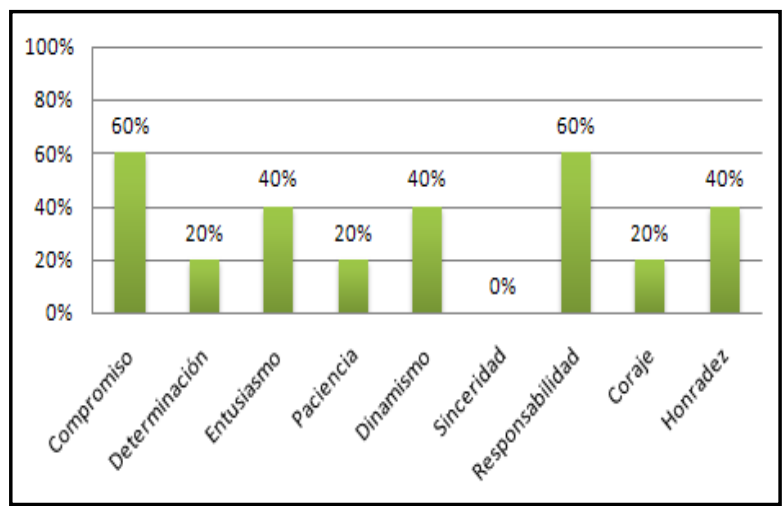

Fuente: DisFruta - Huancayo.

Elaboración: Propia

Como se aprecia en el Figura $\mathrm{N}^{\circ} 5$, las actitudes más sobresalientes entre las colaboradoras de DisFruta - Huancayo, son las de compromiso y responsabilidad, que tienen como resultado un $60 \%$, se puede inferir que el personal desarrolla su labor haciéndose responsable de sus actos, entendiendo que su buena o mala disposición por realizar sus actividades influye en los resultados. Nótese también que no hubo alguna colaboradora que tenga la actitud de la sinceridad, y esto puede influye identificación del equipo humano.

FIGURA N ${ }^{\circ} 6$

Distribución porcentual sobre las habilidades que posee el personal de DisFruta Huancayo

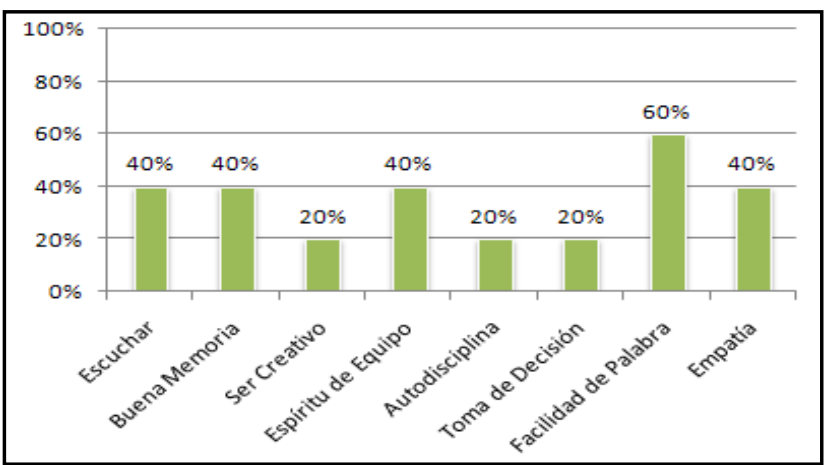

Fuente: DisFruta - Huancayo.

Elaboración: Propia

Como se aprecia en el Figura $\mathrm{N}^{\circ} 6$, se disgrega 8 diferentes habilidades, dentro de ellas la que ocupa el primer lugar es la de facilidad de palabra teniendo un $60 \%$, DisFruta - Huancayo se dedica a la venta de jugos y zumos, es preciso que las colaboradoras tengan facilidad de palabra, ya que así podrán tener poder de convencimiento y de esta manera logren persuadir al cliente. La facilidad de palabra muchas veces es innata, y otras veces se forma, lo cierto es que el criterio tiene un rol determinante en que la facilidad de palabra se convierta en poder de convencimiento.

A diferencia del Figura $N^{\circ} 5$, todas las habilidades quedaron ocupadas por las colaboradoras, lo que no ocurrió con las actitudes.

Es por ello que el $20 \%$ respectivamente lo ocupan; ser creativo, autodisciplina y toma de decisión.
FIGURA No 7

Necesidades insatisfechas

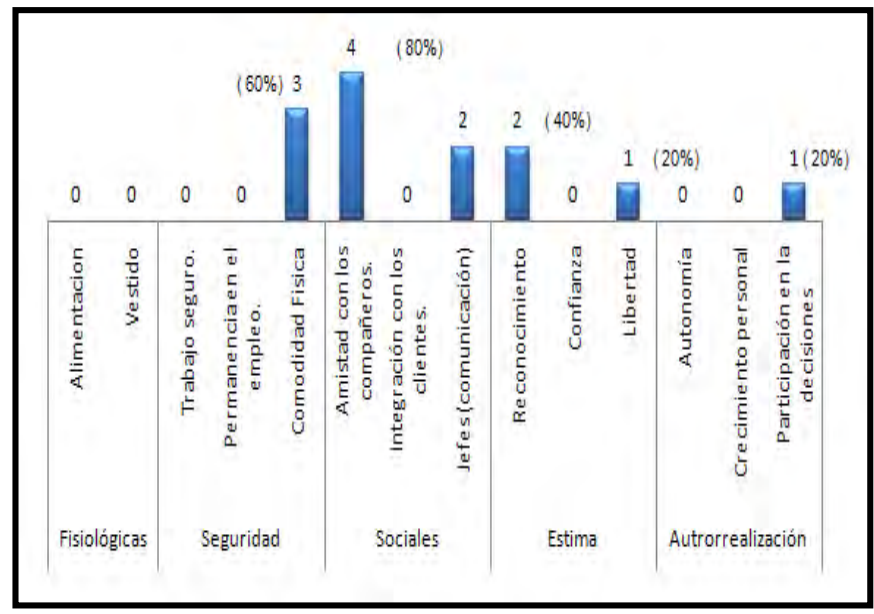

Basado en: Idalberto Chiavenato (2009). Comportamiento Organizacional.

Elaboración: Propia

La Gráfico №7, busca mostrar el ranking de las necesidades que no fueron satisfechas, así se puede apreciar, que la especificación amistad con los compañeros, ocupa el primer lugar teniendo a cuatro colaboradoras que muestran su insatisfacción, este $80 \%$ aseguró que no tienen una buena amistad entre ellas, lo cual hace que la identificación del equipo humano se vea resquebrajado.

Por otro lado se observa la comodidad física, que ocupa el segundo lugar con 3 colaboradoras que expresan su insatisfacción, este $60 \%$ afirmó que el espacio físico de DisFruta - Huancayo, es muy pequeño para el numero de colaboradoras, lo que logran que ellas mismas cambien de carácter y se torne en un clima organizacional negativo.

El tercer lugar ocupan las especificaciones; jefes (comunicación) y reconocimiento, cada uno con dos colaboradoras pronunciando su insatisfacción, estos $40 \%$ dieron a conocer que la comunicación con sus jefes y el reconocimiento no es como quisieran, existen pequeños favoritismos por una u otra colaboradora.

Y por último, el cuarto lugar lo ocupan las especificaciones; libertad y participación en las decisiones, nótese que una colaboradora por cada especificación están mostrando su insatisfacción que equivale a un $20 \%$ respectivamente, en cuanto a la libertad, es por el poco espacio físico que cuenta el establecimiento de DisFruta- Huancayo, y en materia a participación en las decisiones, es por el favoritismo ya mencionado y por la poca motivación laboral. Concluido el proceso de evaluación de las necesidades, se determino los elementos motivacionales por necesidad, a continuación la Figura $\mathrm{N}^{\circ}$ 8, que detalla que tipo de motivación se realizara por necesidad insatisfecha

FIGURA N8

Determinación de elemento motivacional

\begin{tabular}{|ll|}
\hline NECESIDAD & ELEMENTO \\
\hline MOTIVACIONAL \\
\hline Seguridad & Material \\
\hline Social & Mixto \\
\hline Estima & Mixto \\
\hline Autorrealización & Mixto \\
\hline
\end{tabular}

Fuente: DisFruta - Huancayo.

Elaboración: Propia 
En cuanto a la Necesidad de Seguridad, se converso con la administración de DisFruta - Huancayo, para ayudarlos a rediseñar sus tareas por colaborador, de manera tal de organizar funciones y evitar la saturación en determinadas horas por el poco espacio físico.

En cuanto a las demás necesidades, el elemento motivación fue mixto, ya que era una mezcla de lo material y no material que básicamente son los momentos, esos pequeños detalles que agrandan la vida, que se esconden en algún instante del tiempo, esperando listos para ser. $Y$ es que muchas veces en las empresas queda poco tiempo para conocerse como personas, la rutina de trabajar logra que no seamos nosotros dentro de nuestro centro laboral.

Se busco realizar actividades, tales como; premiar a la mejor colaboradora del mes, celebrar los cumpleaños por meses. Así también se realizo evoluciones de $360^{\circ}$ y $180^{\circ}$, a modo tal de evaluar a toda la empresa y detectar errores y solucionarlos.

Después de haber realizo este último paso de la segunda parte del Modelo Aplicativo, se evaluó cognoscitivamente al personal, para saber si se ha solucionado los empirismos aplicativos, los resultados fueron favorables.

Asimismo se evaluó por segunda vez las necesidades de las colaboradoras, esta vez los resultados fueron positivos estaba resuelto el problema, estaba satisfecha sus necesidades

De esta manera se logro solucionas los empirismos aplicativos, carencias en la identificación del equipo humano y por efecto la deficiencia en las actividades

\section{RESULTADOS OBTENIDOS DEL MODELO APLICATIVO}

a. Se comprobó que la motivación laboral en DisFruta Huancayo, fue afectada por Empirismos aplicativos, Carencias y Deficiencias; que influyeron negativamente en el clima organizacional de la empresa.

b. Se logro satisfacer las necesidades de la pirámide de A.Maslow al $100 \%$

c. Las colaboradoras empezaron a mostrar respeto por las disposiciones de sus superiores.

d. El personal llegó a conocer los conceptos adecuados sobre los temas de Clima Organizacional, Motivación y Personalidad.

e. La Motivación se notó incrementada ya que se involucraron las colaboradoras mas con la empresa en temas de participación activa.

f. El diseño del modelo aplicativo ha permitido establecer una eficiente secuencia de pasos para lograr los objetivos.

\section{CONCLUSIONES}

a) La motivación laboral en DisFruta - Huancayo, fue afectada por Empirismos aplicativos, Carencias y Deficiencias; que influyeron negativamente en el clima organizacional de la empresa

b) Dentro de DisFruta - Huancayo las colaboradoras no mostraban un respeto o identificación por las Disposiciones Gerenciales, no se conocían o aplicaban mal algunas de las técnicas avanzadas, y no se habían adecuado al contexto interno para obtener los mayores beneficios potenciales, todo ello explica y está relacionado causalmente con los empirismos aplicativos, que afectaban negativamente al logro de los objetivos de DisFruta - Huancayo.

c) El hecho que las colaboradoras no hayan respetado las políticas laborales y disposiciones gerenciales, no hayan conocido o aplicado mal algunos conceptos, todo lo anterior explica y está relacionado causalmente con las carencias de la identificación del equipo humano, que afectaban negativamente a la motivación laboral y su influencia con el clima organizacional de DisFruta - Huancayo d) Las actividades laborales desarrolladas en DisFruta Huancayo, tenían deficiencias; debido a que no se respetaban las disposiciones gerenciales, o no se aplicaban correctamente las técnicas avanzadas, o no realizaban eficientemente sus actividades laborales las colaboradoras.

e) Se corroboró la utilidad del Marco Teórico, ya que fueron de mucha utilidad para direccionar mejor la presente investigación, conceptos que a su vez cooperaron en la elaboración del Modelo Aplicativo, probando así su utilidad.

f) Se evidenció que el Modelo Aplicativo generado a partir de la Teoría de Necesidades de Abraham Maslow, sirvió para solucionar los problemas de DisFruta - Huancayo, logrando de esta manera una motivación adecuada y un clima organizacional positivo.

g) La nueva realidad que se observa en DisFruta Huancayo, es el reflejo de una ordenada ejecución de proceso de elaboración de la presente investigación, aquella que ha logrado cumplir sus objetivos.

\section{VII.- RECOMENDACIONES}

a) Continuar con los esfuerzos motivacionales, a modo de obtener una mejora continua, la misma que garantizará colaboradoras motivadas y se verá reflejada en el clima organizacional. Logrando así resolver los empirismos aplicativos, carencias en la identificación del equipo humano y deficiencias en las actividades laborales

b) Realizar capacitaciones continuas, a fin de que se conozcan y apliquen mejor las técnicas avanzadas, logrando que las colaboradoras se adecuen al contexto interno y puedan obtener los mayores beneficios potenciales, que beneficiara a DisFruta Huancayo, ya que las colaboradoras realizarán mejor sus actividades conociendo la importancia de la motivación laboral en el clima organizacional.

c) Seguir evaluando cada cierto tiempo la identificación del equipo humano, mejorando continuamente la unión y el trabajo en equipo entre las colaboradoras. También seguir premiando a la mejor colaboradora del mes y celebrando los cumpleaños por mes.

d) Evaluar constantemente la eficiencia de las Actividades Laborales, que ayudara para saber si nuevamente están existiendo empirismos aplicativos y carencias en la identificación del equipo humano, ya que estos dos problemas anteriores son los que causan la deficiencia en las actividades laborales en DisFruta - Huancayo.

e) Recurrir a las teorías vertidas en la presente investigación, que son referentes influyentes en las investigaciones que se realizan sobre Administración de Empresas y Recursos Humanos. Aquellas que ayudarán, guiarán y lograrán contribuir al Marco Teórico existente acerca de motivación, clima organizacional, la personalidad, inteligencia emocional en el desempeño laboral, satisfacción laboral y demás.

f) Utilizar el Modelo Aplicativo constantemente, a fin de evaluar al personal de DisFruta - Huancayo, y observar el cambio positivo o negativo, el presente Modelo Aplicativo ha logrado solucionar los problemas de la empresa, por ende es importante utilizarlo para lograr una mejora continua, cuidando siempre el capital humano, para que de esta manera se tenga resultados favorables para DisFruta - Huancayo.

g) La presente investigación debe continuar, se sugiere que a partir de ella, nuevos investigadores e interesados en el tema y en la empresa, puedan proseguir con ella, profundizando la realidad y abordando los problemas desde otras perspectivas académicas y metodológicas, ya que la misión 
académica que uno tiene, es la de generar nuevos conceptos acorde a la realidad, así como también recordar que una vez hecha la investigación, ésta se debe publicar, y así difundir y conseguir la innovación del conocimiento, logrando cooperar con nuestra sociedad. Teniendo en cuenta que sólo se podrá tomar la presente investigación como referente, ya

\section{VIII.- REFERENCIAS}

\subsection{REFERENCIAS BIBLIOGRÁFICAS}

a. Caballero Romero Alejandro. Innovaciones en las guías metodológicas para los planes y tesis de maestría y doctorado. Perú. . Editorial Alen Caro.2da Edición.2009

b. Chiavenato Idalberto .Comportamiento Organizacional, la dinámica del éxito en la organizacionaes.2da Edición. Editorial México. Mc Graw- Hill Inteamericana.2009

c. Davis Keith. Newstrom Comportamiento Humano en la Trabajo. Mexico,Editorial Mc Graw Hill.2003

d. Kast Fremont,Rosenzweig James(1985). Administración en las organizaciones. México. Editorial. Mc Graw - Hill.

e. Robbins Stephen. Teoría de la Personalidad. 5ta Edición. México. Editorial Pearson Education. 1998

f. Sigmund Freud. Introducción al Psicoanálisis .1ra Edición. Editorial Alianza.2011.

\subsection{REFERENCIAS ELECTRÓNICAS}

que su solución presentada es única y exclusivamente para DisFruta - Huancayo.

\section{AGRADECIMIENTOS}

Al Ing. Miguel Fernando Inga Ávila y al Lic. Sergio Gamarra Cisneros

a. Inteligencia Emocional en la empresa y el trabajo.

Disponible en: http://www.inteligencia-

emocional.org/trabajoyempresa/index.htm

b. Influencia de la inteligencia emocional en el desempeño laboral. Disponible en: http://www.emagister.com/web/search/?action=searc h\&origen=buscador home\&esBusquedaUsuario $=1 \& \mathrm{q}$ =inteligencia+emocional\&btnBuscar=

c. Inteligencia Emocional; 2007. Disponible en; http://www.gestiopolis.com/dirgp/rec/ie.htm

d. Psicologimante hablando. Disponible en: http://www.psicologicamentehablando.com/personalid ades-tipo-a-y-b/

e. Test de personalidad. Disponible en: http://www.psicoactiva.com/tests/test9.htm

e-mail: sgamarra.sf@gmail.com jpovesvarillas@gmail.com 